\title{
Need to define patient engagement in research
}

$\mathrm{T}$ he federal government has ponied up with the cash and researchers want more patient engagement in health research, but defining what this means - and developing a framework for implementing it - is an ongoing process.

Patient engagement in research, which can include everything from consulting with patients on how to design trials to tailoring research to patient needs, is fostered by the Canadian Institutes of Health Research (CIHR) Strategy for PatientOriented Research (SPOR) program. Since 2011, it has been allocated $\$ 60.5$ million. SPOR provides partial funding for provincial and territorial Support for People and Patient-Oriented Research and Trials (SUPPORT) Units, with the remainder coming from ministries of health, provincial health research organizations, and in some cases, health authorities, academic institutions and industry.

So far, every province has established a SUPPORT Unit, except Saskatchewan and British Columbia; the territories are at different stages of development. But interpreting how to implement SPOR's patient-engagement initiative differs among these units.

"I think folks across the country are still trying to feel their way a little bit as to what it means to meaningfully engage patients and conduct patient-oriented research," says Dr. Mark Roseman, the director of Ontario's SUPPORT Unit, which opened in March 2014 with $\$ 111.7$ million in funding from SPOR and the province. Roseman says engaging patients involves a bit of a culture change, and the centre is still developing the science behind it.

A common concern is the fear of involving patients in research in only a token way. Dr. Neil Andersson, director of the Participatory Research at McGill (PRAM) in Montréal, says "We have a real issue with the cardboard cut-out of participation where you designate someone, often fairly articulate, and almost always someone pretty smart, to be the patient representative." PRAM began

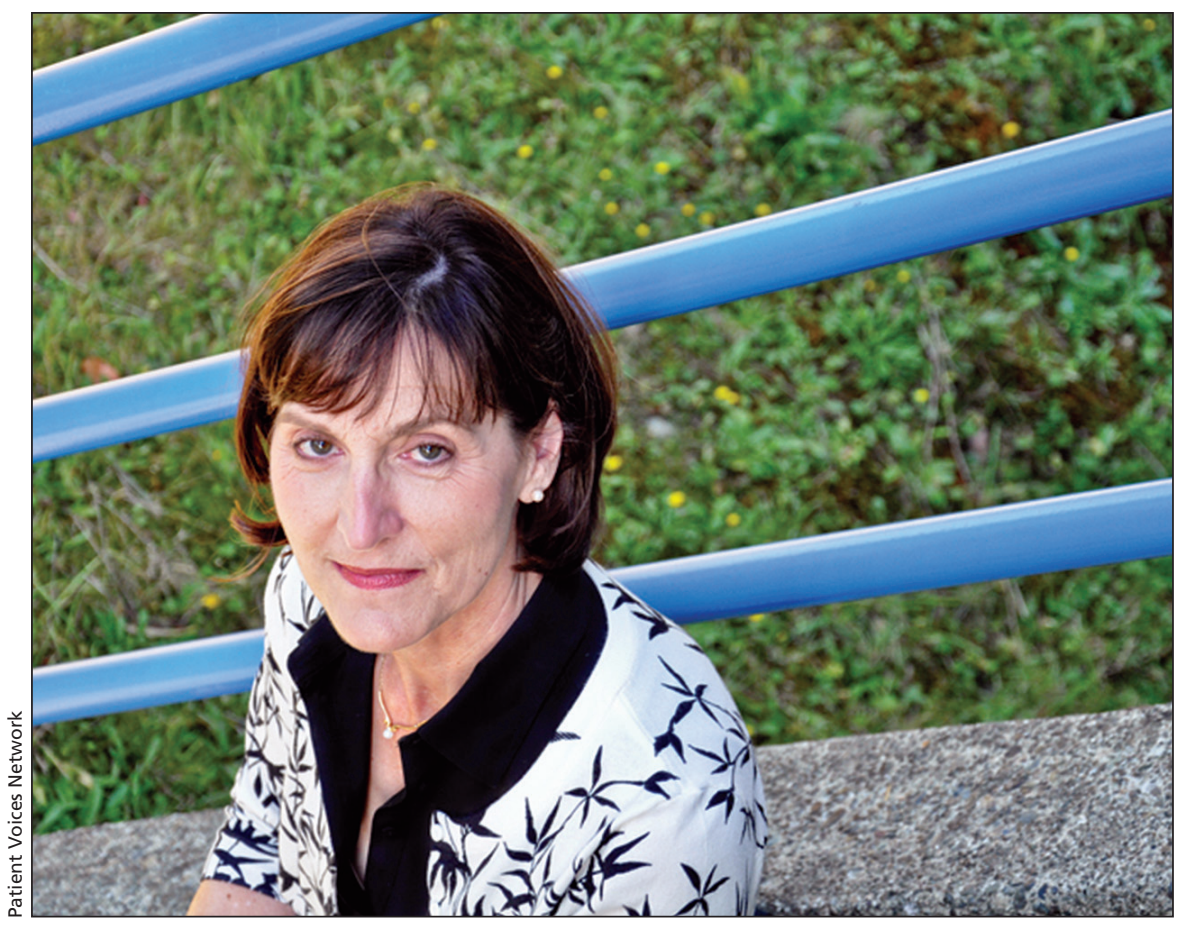

Colleen McGavin is offering her perspective as a patient to researchers in British Columbia.

working with the public in 2006 with community-led randomized controlled trials to study different approaches to complex issues like suicide.

CIHR is aware of potential tokenism and has plans to mitigate it. SPOR's manager Nancy Mason MacLellan says, "meaningful engagement" is not just having a patient on a committee. SPOR intends to "monitor how recipients of CIHR funding are engaging patients not only to ensure that it is occurring, but also to understand how it is being done."

Some researchers have found other tactics to avoid tokenism. Dr. Andreas Laupacis, executive director of the $\mathrm{Li}$ Ka Shing Knowledge Institute of St. Michael's Hospital, uses the James Lind Alliance approach. It involves patients, caregivers and researchers equally in a committee that identifies research priorities. In his experience with patients, he says "one thing that pisses them off more than not being involved is being involved and being ignored."

More grants are also being awarded to researchers who have a patient focus.
Dr. Susan Kahn, associate director for clinical research at the Lady Davis Institute in Montréal, says this is a positive move. "Why should it be up to researchers who have no experience personally with a particular disease to decide what a study design should look like or what outcomes should be measured?"

Another potential pitfall is the patient's lack of impartiality. Dr. Donald Redelmeier, a senior scientist at the Sunnybrook Research Institute in Toronto, says "No patient can be impartial. There are all sorts of conscious and unconscious bias." These may include biases in favour of studying a disease or treatment.

Complicating matters further is the lack of evidence about patient engagement in research; some of the little research that exists questions its usefulness. The United Kingdom adopted patient engagement in research in 1996, but a qualitative study in BMJ Open in December 2014 indicates there is little evidence informing the implementation of these programs. A June 2014 article 
in the same journal reported that tokenism is common. But that article also concluded that with proper guidance and measurable standards, public involvement can be valuable.

Over the next few years, SPOR plans to measure the impact of Canada's patient-focused initiatives.

The United States is also venturing into this territory through its PatientCentered Outcomes Research Institute (PCORI).

While doctors, policy-makers and researchers try to define patient roles, the patients themselves are negotiat- ing their position. Since 2010, patient and caregiver Colleen McGavin has volunteered with the BC Patient Voices Network, which has trained over 700 patients to inform the Ministry of Health about patient priorities in research.

McGavin is also helping to establish BC's SPOR Unit, which she views as a way to facilitate research by and with patients, instead of just treating patients as study subjects. "It's giving patients a real voice about what should be researched based on what's important to them."

McGavin was treated for bladder can- cer and participated in a clinical trial as a "last ditch effort" to avoid having a cystectomy. The treatment didn't work for her. She has since inspired a trial on giving women the option to use antibiotics to treat uncomplicated bladder infections, and possibly prevent the antibiotic resistance she developed.

Engagement, she says, is a broad term. "We're at a point in time where this whole process is neither well understood nor well accepted," said McGavin. — Shannon Lough, CMAJ

CMAJ 2015. DOI:10.1503/cmaj.109-5109 